\title{
In Situ Electron Microscopy Investigation of Sodiation of Titanium Disulfide Nanoflakes
}

Xiuzhen Wang, ${ }^{\dagger, \$ ;}$ Zhenpeng Yao, ${ }^{\#, i}$ Sooyeon Hwang, ${ }^{\ddagger}$ Ying Pan, ${ }^{\natural}$ Hui Dong, ${ }^{\perp}$ Maosen Fu, ${ }^{\S} \mathrm{Na}$

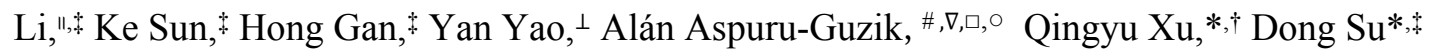

${ }^{\dagger}$ School of Physics, Southeast University, Nanjing 211189, China

*Center for Functional Nanomaterials, Brookhaven National Laboratory, Upton, New York 11973, United States

\#Department of Chemistry and Chemical Biology, Harvard University, Cambridge, MA, 02138 USA

'Department of Materials Science \& Engineering, Massachusetts Institute of Technology, Cambridge, Massachusetts, 02139, United States

${ }^{\perp}$ Department of Electrical \& Computer Engineering and Materials Science and Engineering Program, University of Houston, Houston, Texas 77204, United States

\$Shanxi Materials Analysis and Research Center, School of Materials Science and Engineering, Northwestern Polytechnical University, Xi'an 710000, China

"Frontier Institute of Science and Technology jointly with College of Science, State Key Laboratory for Mechanical Behavior of Materials, Xi'an Jiaotong University, Xi'an 710054, China

${ }^{\nabla}$ Department of Chemistry and Department of Computer Science, University of Toronto, Toronto, Ontario M5S 3H6, Canada

-Vector Institute for Artificial Intelligence, Toronto, Ontario M5S 1M1, Canada

${ }^{\circ}$ Canadian Institute for Advanced Research (CIFAR) Senior Fellow, Toronto, Ontario M5S 1M1, Canada 


\section{Supporting Movie Showing the Structural Evolution of the $\mathrm{TiS}_{2}$ upon Na Insertion}

(1). Movie S1: In situ SAED during the whole sodiation process of $\mathrm{TiS}_{2}$ corresponding to the Figure S3.

(2). Movie S2: In situ SAED during the intercalation process of $\mathrm{TiS}_{2}$ corresponding to the Figure S4.

(3). Movie S3: In situ STEM during the intercalation process of $\mathrm{TiS}_{2}$ corresponding to the Figure 3(a).

(4). Movie S4: In situ STEM during the whole sodiation process of $\mathrm{TiS}_{2}$ corresponding to the Figure 4(a).

\section{First-Principles Calculations}

Identify the ground state structures of the $\mathrm{Na}_{x} \mathrm{TiS}_{2}(x=0.33,0.55)$ phases

The lowest-energy, ground state structures of $\mathrm{Na}_{x} \mathrm{TiS}_{2}(x=0.33,0.55)$ are determined by a vast number of geometrically-distinct $\mathrm{Na} / \mathrm{Vac}$ ordered configurations using DFT calculation based NEPS method. ${ }^{1,2}$ Starting from the partially-occupied structure from the XRD refinement, which has $\mathrm{Na}$ /vacancy sharing the $\mathrm{Na}$ sites as shown in Figure S9 and S10, we generate supercells containing $6 \mathrm{Na}$ sites and $9 \mathrm{Na}$ sites respectively. We then populate the $\mathrm{Na}$ sites with $\mathrm{Na}$ and vacancy in the ratio 1:2. 64 and 546 geometrically different configurations were generated using the Enum code. ${ }^{3-5}$ We calculated the electrostatic total energy for all configurations using nominal charge states for the ions in the system as a quick energy sampling step. ${ }^{6}$ All structures were ranked by their normalized electrostatic energies, and the $50 \mathrm{Na}_{0.33} \mathrm{TiS}_{2}$ and 100 $\mathrm{Na}_{0.55} \mathrm{TiS}_{2}$ structures with the lowest electrostatic energies were fully relaxed, and their 
energies calculated, using DFT. We find the structure with the lowest DFT total energy, i.e., the ground state structures of $\mathrm{Na}_{0.33} \mathrm{TiS}_{2}$ and $\mathrm{Na}_{0.55} \mathrm{TiS}_{2}$ have space groups of $C 2 / m$ (Figure S9) and P1 (Figure S10).

The $2 \mathrm{D}$ to $3 \mathrm{D}$ transition occurs along with the sodiation mechanism switching from intercalation to extrusion. After a full intercalation to $\mathrm{NaTiS}_{2}$, further sodiation may partially destroy the Ti-S slabs which is the backbone of this layered structure, and induce an extrusion reaction. From the energy point of view, assuming the 2D-3D transition happened at composition of $\mathrm{Na}_{x} \mathrm{TiS}_{2}$ and the phase transition follows the formula of $\mathrm{Na}_{x} \mathrm{TiS}_{2} \rightarrow \mathrm{Na}_{2} \mathrm{~S}+\mathrm{Ti}_{2} \mathrm{~S}$. Before the reaction, the free energy is:

$$
\Delta G_{\text {before }}=V_{0} G\left(\mathrm{Na}_{x} \mathrm{TiS}_{2}\right)+S_{2 \mathrm{D}} f_{2 \mathrm{D}}
$$

where $G\left(\mathrm{Na}_{x} \mathrm{TiS}_{2}\right)$ is the Gibbs free energy per unit volume of the before reaction phase; $V_{0}$ is the volume of particle; $S_{2 \mathrm{D}}$ is the surface area of particle in before reaction and $f_{2 \mathrm{D}}$ is the surface energy per unit area of before reaction. After the reaction, the free energy is

$$
\Delta G_{\mathrm{after}}=V_{1} G\left(\mathrm{Na}_{2} \mathrm{~S}\right)+V_{2} G\left(\mathrm{Ti}_{2} \mathrm{~S}\right)+S_{i} f_{i}+S_{3 \mathrm{D}} f_{3 \mathrm{D}}
$$

where $G\left(\mathrm{Na}_{2} \mathrm{~S}\right)$ and $G\left(\mathrm{Ti}_{2} \mathrm{~S}\right)$ are the Gibbs free energy per unit volume after reaction phase; $V_{1}$ and $V_{2}$ are respectively the volume of two phases after reaction; $S_{i}$ and $f_{i}$ are respectively the interface area and interface energy between $\mathrm{Na}_{2} \mathrm{~S}$ and $\mathrm{Ti}_{2} \mathrm{~S}$ phase; $S_{3 \mathrm{D}}$ and $f_{3 \mathrm{D}}$ are respectively surface area and surface energy in after reaction phase. $\Delta G_{A}-\Delta G_{B}$ is the driving force for such a transition. The main contribution to the driving force comes from phase separation. The second contribution is from decreasing surface energy because of decreasing surface area from $2 \mathrm{D}$ to $3 \mathrm{D}$. However, the 
decreasing free energy of the two parts needs to overcome the increasing interface energy. The negative value of the driving force can lead to the reaction occurrence. In addition, the transition from $2 \mathrm{D}$ to $3 \mathrm{D}$ may require remarkable mass diffusion, which can be an important factor to determine the reaction kinetics.

\section{REFERENCES}

(1). Yao, Z.; Kim, S.; He, J.; Hegde, V. I.; Wolverton, C. Interplay of Cation and Anion Redox in $\mathrm{Li}_{4} \mathrm{Mn}_{2} \mathrm{O}_{5}$ Cathode Material and Prediction of Improved $\mathrm{Li}_{4}(\mathrm{Mn}, \mathrm{M})_{2} \mathrm{O}_{5}$ Electrodes for Li-Ion Batteries. Sci. Adv. 2018, 4, eaao6754.

(2). Yao, Z.; Kim, S.; Aykol, M.; Li, Q.; Wu, J.; He, J.; Wolverton, C. Revealing the Conversion Mechanism of Transition Metal Oxide Electrodes during Lithiation from First-Principles. Chem. Mater. 2017, 29, 9011-9022.

(3). Hart, G. L. W.; Forcade, R. W. Algorithm for Generating Derivative Structures. Phys. Rev. B 2008, 77, 224115.

(4). Hart, G. L. W.; Forcade, R. W. Generating Derivative Structures from Multilattices: Algorithm and Application to Hcp Alloys. Phys. Rev. B 2009, 80, 014120.

(5). Hart, G. L. W.; Nelson, L. J.; Forcade, R. W. Generating Derivative Structures at a Fixed Concentration. Comput. Mater. Sci. 2012, 59, 101-107.

(6). Michel, K. J.; Zhang, Y.; Wolverton, C. Fast Mass Transport Kinetics in $\mathrm{B}_{20} \mathrm{H}_{16}$ : A High-Capacity Hydrogen Storage Material. J. Phys. Chem. C 2013, 117, 1929519301. 

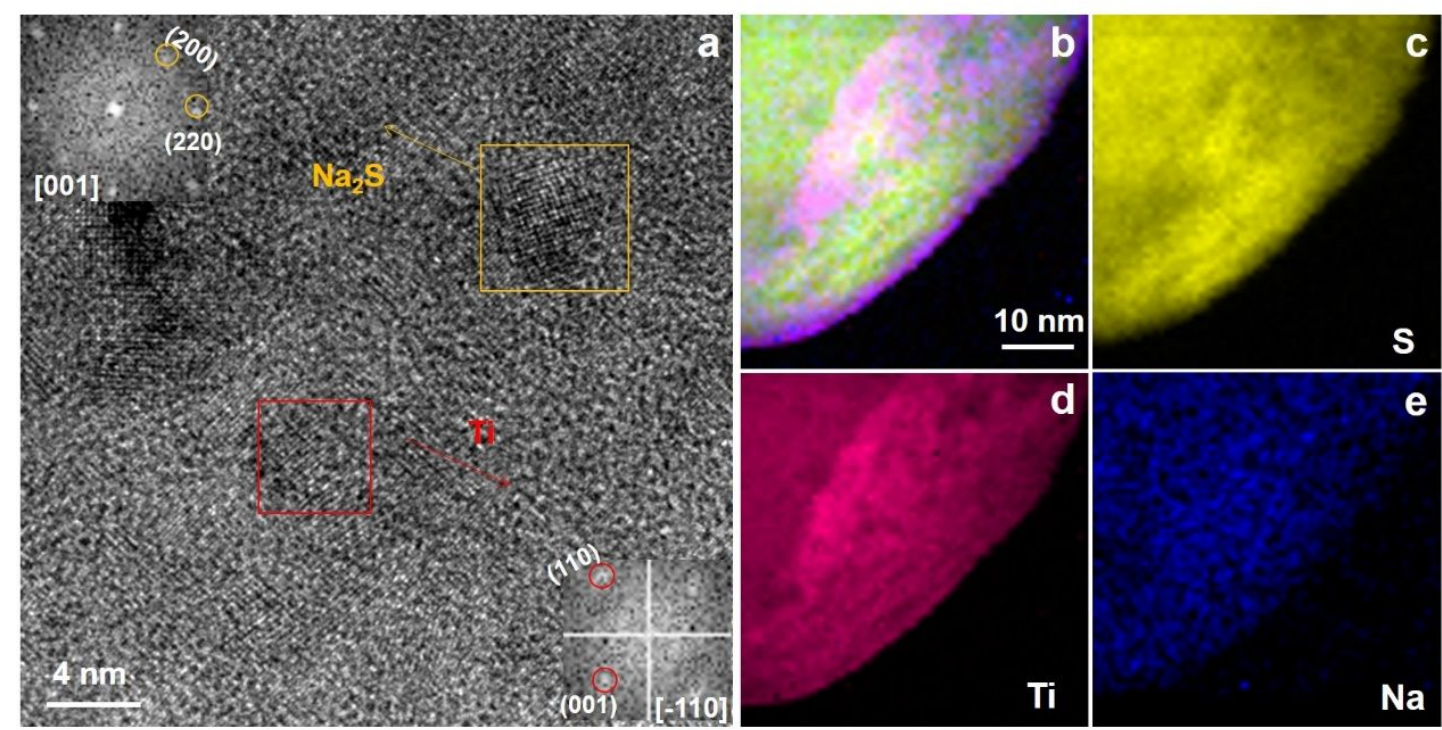

Figure S1. (a) The ex situ HR-TEM of the $\mathrm{TiS}_{2}$ electrode after first discharge to $0.1 \mathrm{~V}$. (b) The corresponding STEM-EELS maps showing the $\mathrm{S} \mathrm{(c),} \mathrm{Ti}$ (d) and $\mathrm{Na}$ (e) distributions, and the mix map (b) indicating the extruded Ti on the surface. 

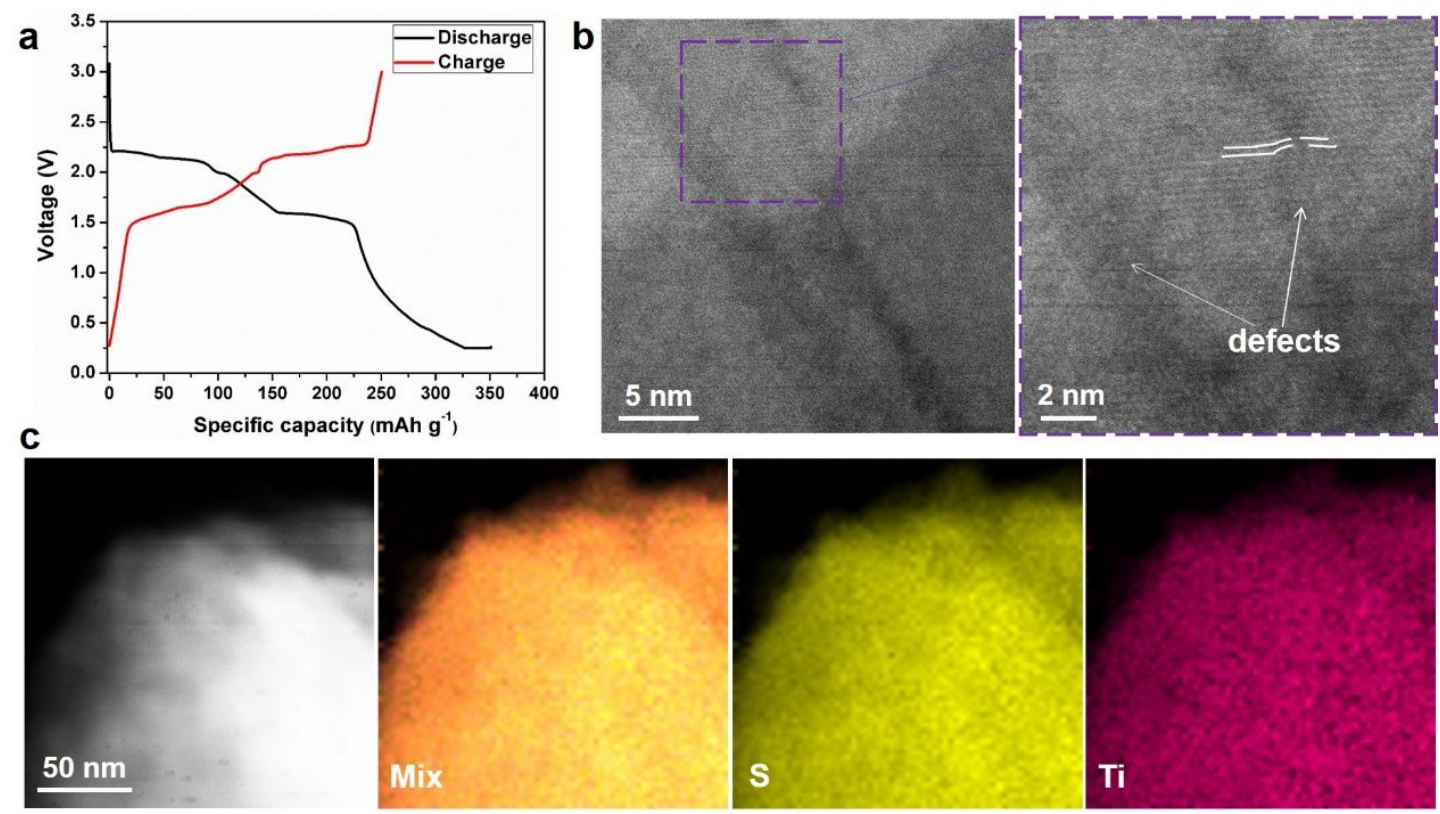

Figure S2. (a) Initial discharge-charge voltage profile of $\mathrm{TiS}_{2}$ electrode in the range of $0.1-3 \mathrm{~V}$. At the charge state, (b) the HAADF-STEM images and (c) the STEM-EELS maps. 


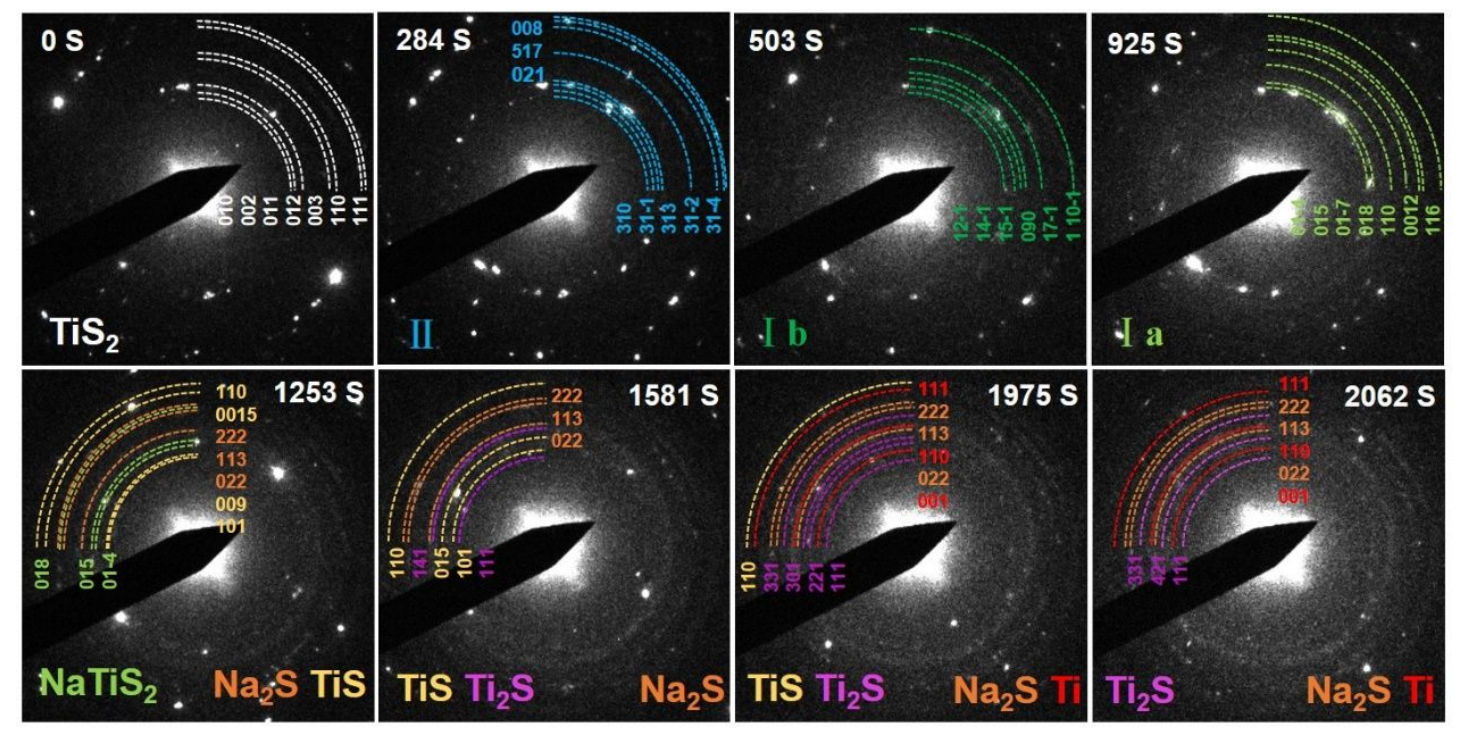

Figure S3. Diffraction patterns from in situ SAED corresponding to different phases in intensity profiles during sodiation. 


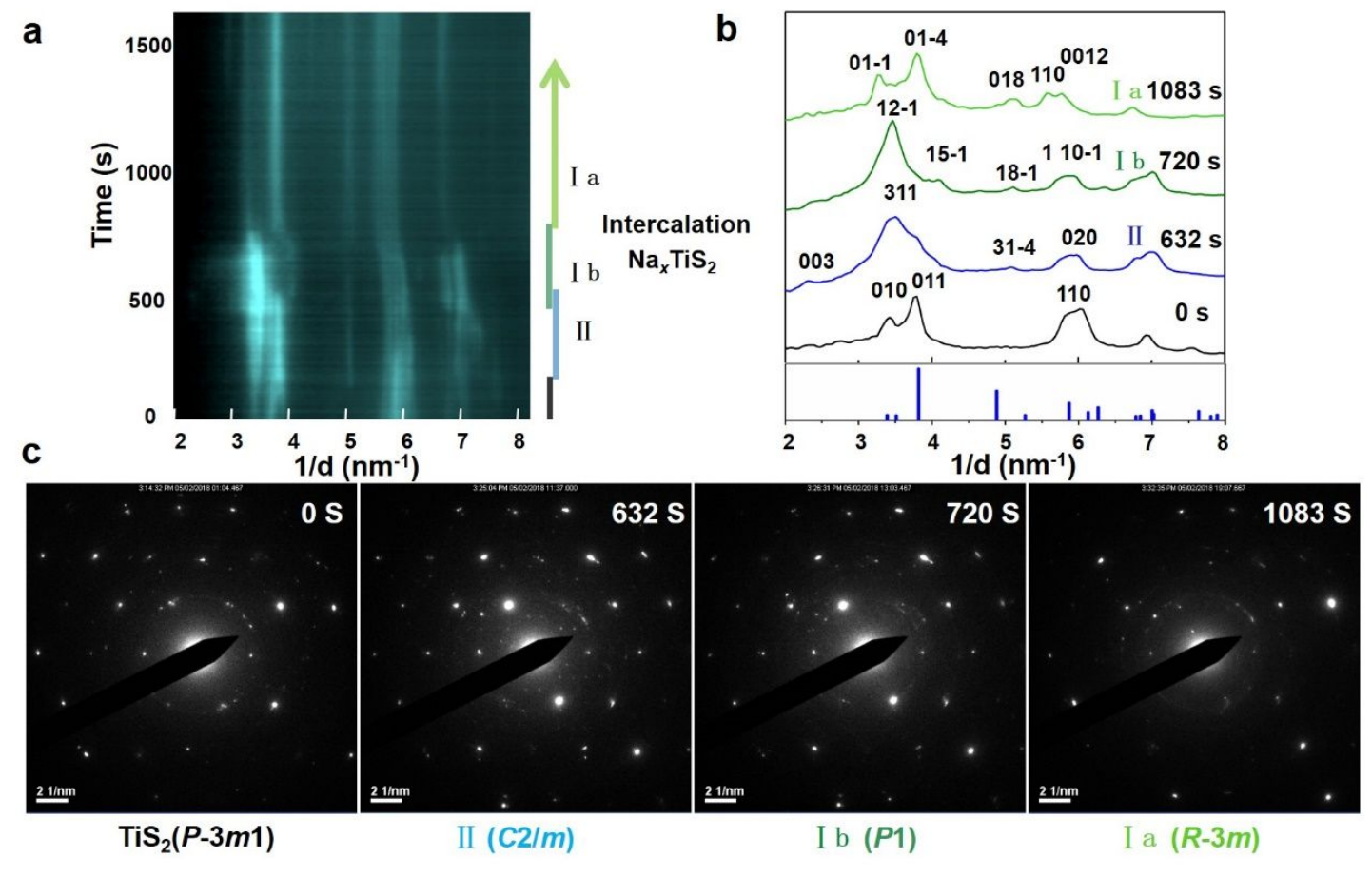

Figure S4. (a) Colored intensity profiles as a function of reaction time during sodiation of $\mathrm{TiS}_{2}$. (b)

Corresponding radially integrated intensity profiles of SAED patterns at certain time. (c) Diffraction patterns correspond to intensity profiles. 

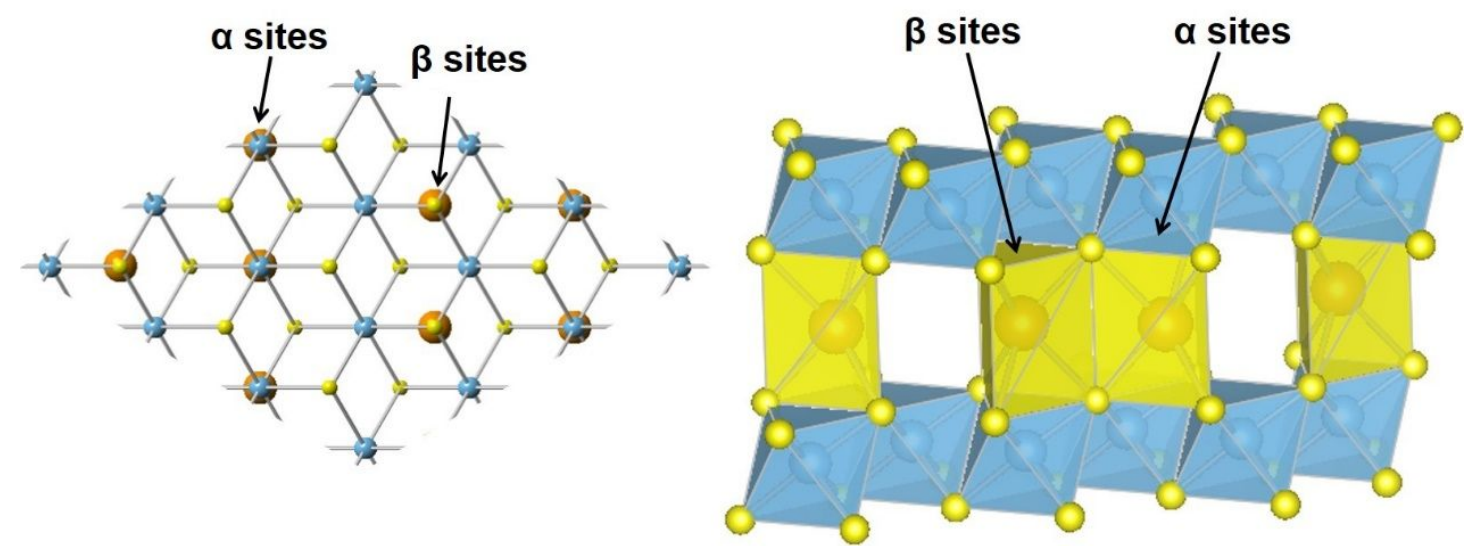

Figure S5. In II phase, the Na ions can occupy one of two symmetrically equivalent trigonal prismatic sites $(\alpha, \beta)$, three-dimensional models of the trigonal prismatic. 


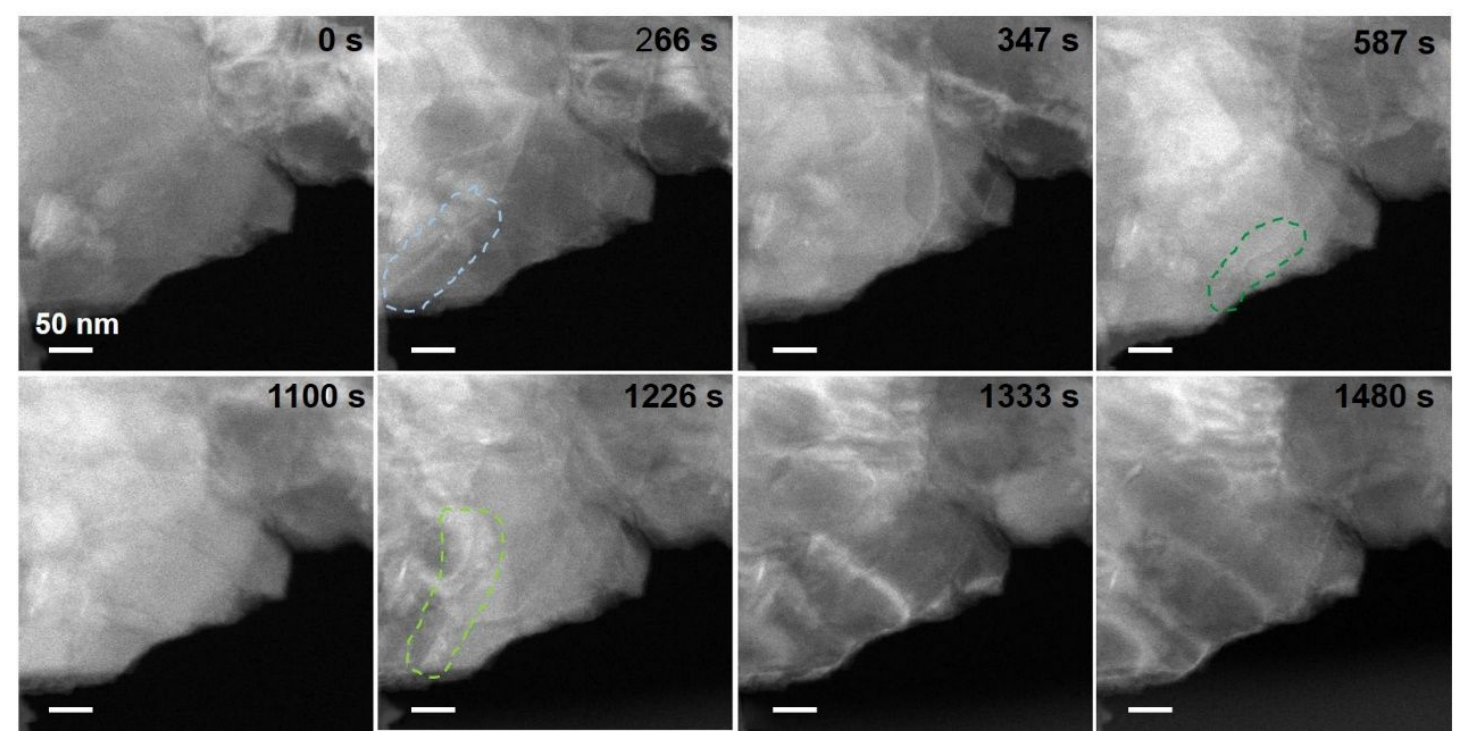

Figure S6. Time-sequence raw dark field images from Movie 3. 


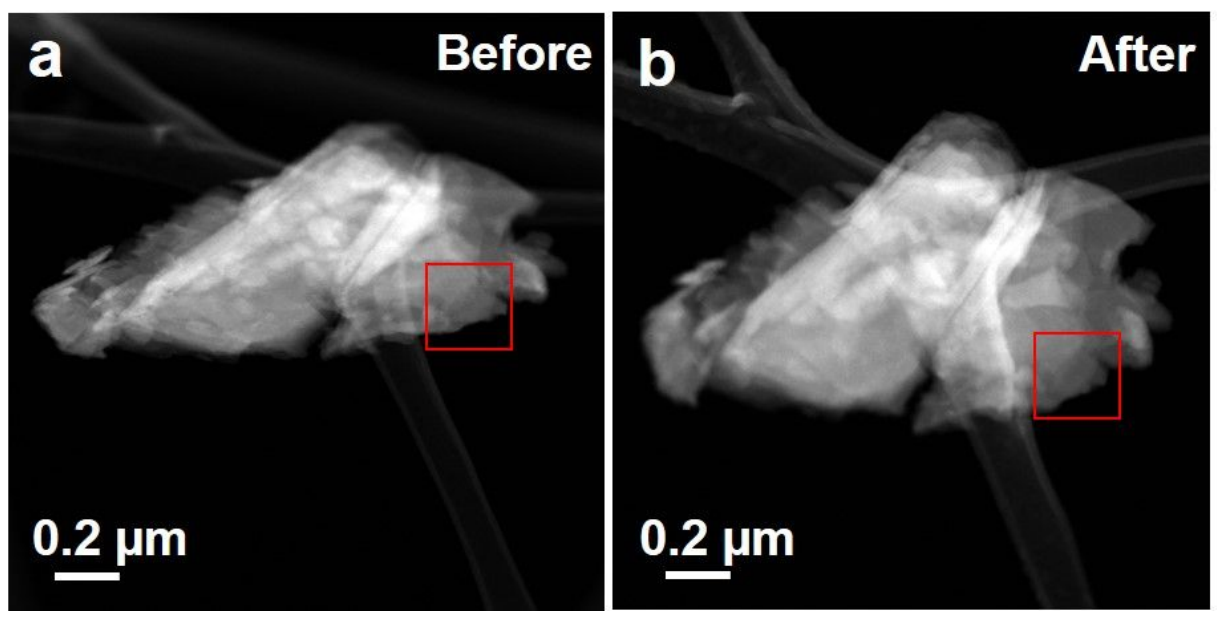

Figure S7. The low-mag STEM-ADF images before (a) and after (b) the reaction. The specific area for

Figure 3 a was marked in a red box. 


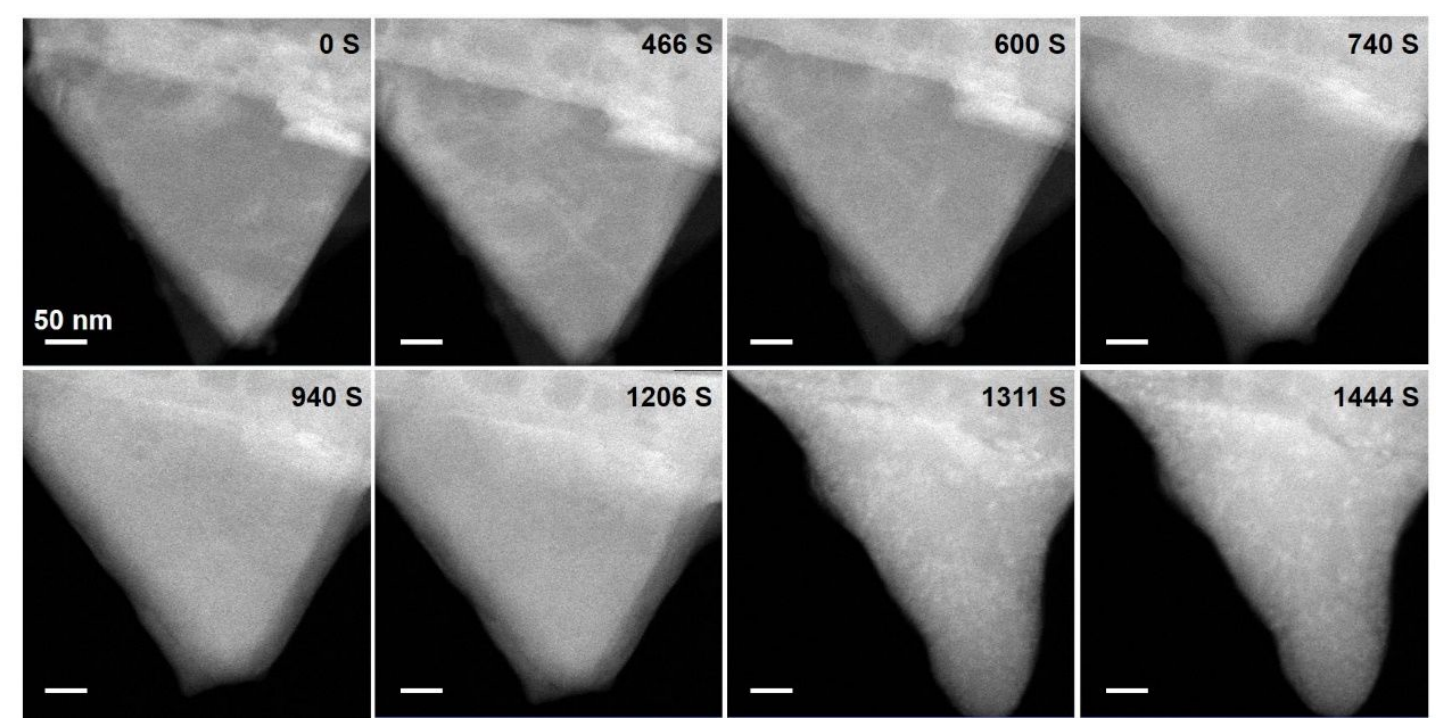

Figure S8. (a) In situ HAADF-STEM images showing phase evolution during sodiation. 


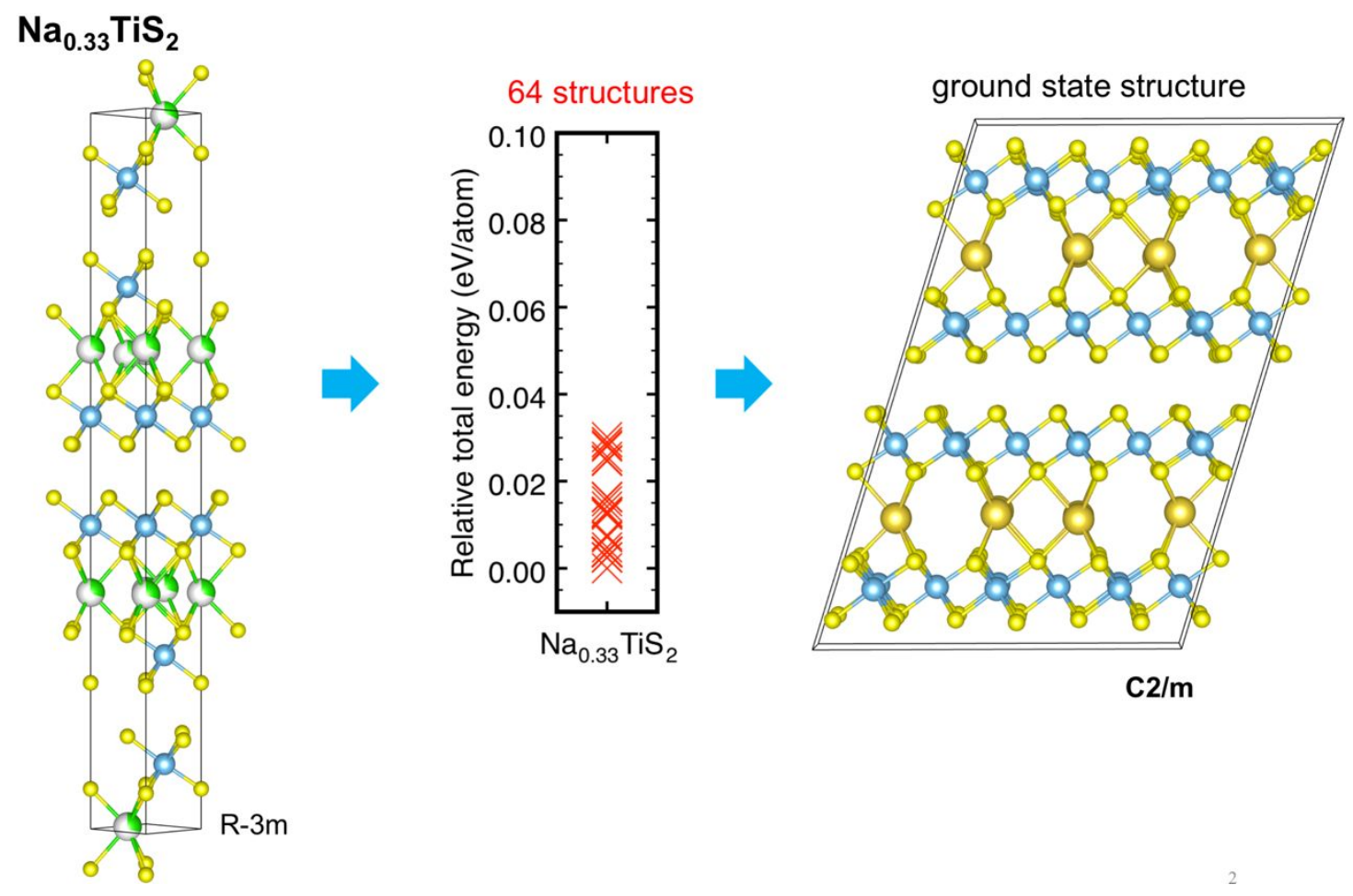

Figure S9. Determine the ground state structure of $\mathrm{Na}_{0.33} \mathrm{TiS}_{2}$. 

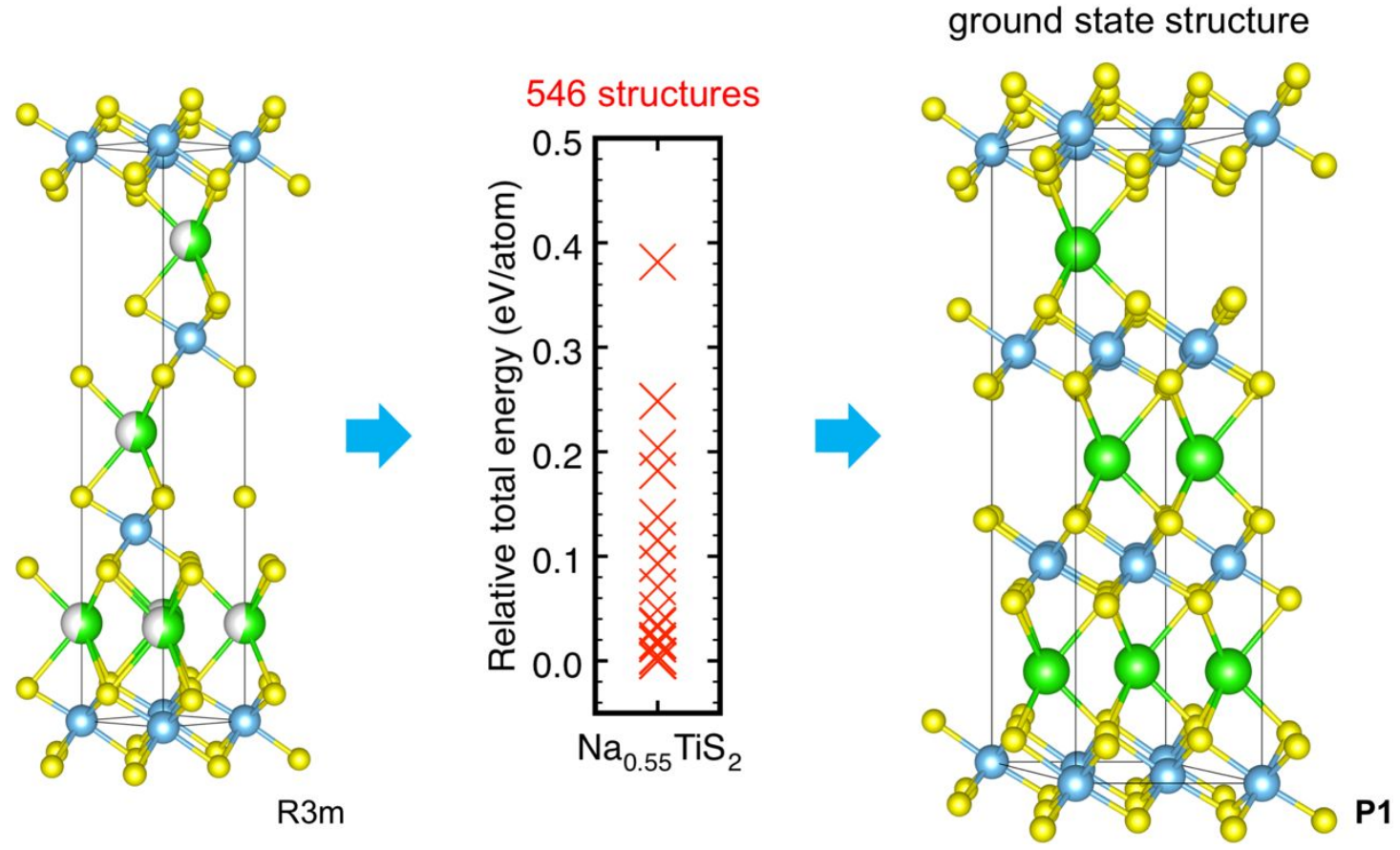

Figure S10. Determine the ground state structure of $\mathrm{Na}_{0.55} \mathrm{TiS}_{2}$. 